\section{THU0547 FIBRODYSPLASIA OSSIFICANS PROGRESSIVA IN PEDIATRIC RHEUMATOLOGY PRACTICE: LARGE SERIES EXPERIENCE OF THE SINGLE CENTER}

Irina Nikishina, Alia Latypova, Maria Kaleda, Svetlana Arsenyeva, Dmitrii Alexseev. Nasonova Research Institute of Rheumatology, Moscow, Russian Federation, Pediatric, Moscow, Russian Federation

Background: Fibrodysplasia Ossificans Progressiva (FOP), also known as a "second skeleton disease" is extremely rare (1: 2000000) and disabling genetic disorder, caused by mutation of ACVR1 gene, a bone morphogenetic protein receptor. Among medical specialties there are no certain, capable to provide not only diagnostics, but also all medical maintenance (assessment and monitoring of extent of damages, the differentiated drug treatment, rehabilitation, contact with adjacent experts). It would be reasonable if the rheumatologist carried out a role of the main attending physician for the patient with FOP. It seems to be a lot of similarities between rheumatic diseases, especially spondyloarthritis $(\mathrm{SpA})$ and FOP in the pathophysiology, clinical manifestation and the therapy approach. Objectives: to present the single-center experience of the FOP patients and to identify similar symptoms in FOP and rheumatic disease.

Methods: The analysis of the large series of patients with FOP, who observed in the rheumatologic clinic.

Results: Our single center experience includes 26 patients with FOP. All 26 patients (13 male and 13 female) had 3 basic FOP clinical manifestations. In 23 patients molecular-genetic tests were performed and typical mutation (Arginine 206) occurred in 22 cases and one had an extremely rare mutation (Glicine 328). 22 (85\%) patients had common for FOP massive heterotopic ossifications. 3 of them had formed heterotopic ossification through the X-ray negative stage to visible changes. Among typical phenotypic stigmas were: great toe malformation; thumbs malformation; peripheral osteochondromas; cervical spine abnormalities. Majority of the cases presented some similarities to SpA manifestations: ankylosis of the apophysial joints and vertebral bodies mostly in cervical spine; X-ray/CT evidence of the sacroilitis in all patients, who were examined $(n=8)$. Because of severe body deformity or metal details after previous surgery intervention there were no possibility to perform MRI in most patients, but we confirm typical sacroiliitis with extended bone edema on MRI in 3 patients. Recurrent episodes of the large joints synovitis were present in 5 patients. 4 patients demonstrated gradually formation of great toe ankylosis during the follow-up observation. The involution and decreasing of new FOP nodes associated with non-steroidal anti-inflammatory drug intake and/or glucocorticoids therapy were occurred in all patients.

\begin{tabular}{|c|c|c|c|c|}
\hline Clinical features & & Male & female & $\begin{array}{l}\text { Total } \mathrm{N} \text { of } \\
\text { patients }\end{array}$ \\
\hline Sex ratio & & 13 & 13 & $26(1: 1)$ \\
\hline Age of initial manifestations & $<1$ & 8 & 1 & $9(35 \%)$ \\
\hline \multirow[t]{2}{*}{ (years) } & $1-9$ & 6 & 8 & $14(54 \%)$ \\
\hline & $\geq 10$ & 0 & 3 & $3(11 \%)$ \\
\hline Great toe malformation & & 13 & 12 & $25(96 \%)$ \\
\hline Thumbs malformation & & 2 & 3 & $5(19 \%)$ \\
\hline Cervical spine abnormalities & & 12 & 9 & $21(81 \%)$ \\
\hline Peripheral osteochondromas & & 8 & 7 & $15(58 \%)$ \\
\hline Massive heterotopic ossifications & & 12 & 10 & $22(85 \%)$ \\
\hline $\mathrm{CT} / \mathrm{X}$-Ray/MRI picture of sacroilitits & & $\begin{array}{l}2 \text { (of } \\
2 \text { ) }\end{array}$ & 6 (of 6) & 8 of $8(100 \%)$ \\
\hline
\end{tabular}

Conclusion: Appearance of the similarities in FOP and SpA manifestation and the therapy approach could identify FOP as a potential rheumatic disease. Clinical observation of FOP patients could provide the important information for rheumatologist about insufficiently explored process of new bone formation.

Disclosure of Interests: None declared

DOI: 10.1136/annrheumdis-2019-eular.8044

\section{THU0548 ASSOCIATIONS BETWEEN: MARKERS OF BONE TURNOVER AND RADIOLOGICAL FINDINGS IN CHILDREN DIAGNOSED WITH JUVENILE IDIOPATHIC ARTHRITIS}

Marta Janicka-Szczepaniak, Krzysztof Orczyk, Elzbieta Smolewska. Medical University of Lodz, Department of Pediatric Cardiology and Rheumatology, Lodz, Poland

Background: Although many children attending pediatric rheumatology departments are diagnosed with low bone mineral density on the basis of clinical imaging, it is difficult to determine which patients have the higher risk of developing osteoporosis as the secondary disease. Recently performed studies involving rheumatological patients have proposed numerous serological indicators for better evaluation of the disease activity. These include markers of bone turnover, among others: bone alkaline phosphatase and osteoprotegerin (which are more specific for bone for mation) and also beta isomerized carboxy terminal telopeptide of type I collagen, also known as Beta-Crosslaps (which is considered as an indicator of bone resorption).

Objectives: The main objective of the study was to evaluate the clinical usefulness of measuring serum concentrations of the selected markers of bone turnover in juvenile idiopathic arthritis (JIA) patients and assess their potential significance in prevention of osteoporosis in these children.

Methods: Study involved 59 children previously diagnosed with JIA (mean age at diagnosis: $9.0 \pm 4.3$ years, mean age at study baseline: $12.7 \pm 3.9$ years). All patients underwent Dual X-Ray Absorptiometry (DXA) examinations in order to assess bone mineral density. Wrist radiographs were also taken for evaluation according to the Steinbrocker classification. The presence of abnormalities in these tests was chosen as the criterion to divide patients into subgroups to perform group comparisons for the serum levels of markers of bone turnover: bone alkaline phosphatase, osteoprotegerin and Beta-Crosslaps.

Results: According to the Steinbrocker classification, 10 (16.9\%) patients were staged as class I or higher. These children had significantly lowe serum levels of bone alkaline phosphatase $(p=0.0333)$ than patients with no radiological changes on wrist radiographs. The non-zero Steinbrocker subgroup had also lower Total Body Less Head Z-Score results $(p=0.010)$ than the remaining patients.

The DXA results, expressed as Total Body Less Head and Lumbar Spine Z-Score, disclosed low bone mineral density in $10(16.9 \%)$ and 12 $(20.3 \%)$ patients, respectively. There were no significant correlations between serum levels of markers of bone turnover and bone minera density measurements. However, bone alkaline phosphatase and osteoprotegerin were negatively correlated with DXA muscle mass $(r=-0.359$ $p=0.040$ for bone alkaline phosphatase, $r=-0.372 p=0.0392$ for osteoprote gerin). Similar correlation was found for bone alkaline phosphatase and DXA fat mass $(r=-0.418 p<0.01)$.

Although Beta-Crosslaps appeared to be the only marker of bone turnover significantly $(p=0.0410)$ associated with Juvenile Arthritis Disease Activity Score 27-Joint Count (JADAS27), it was independent from radio logical findings involved in the analysis. However, serum levels of BetaCrosslaps were higher in patients evaluated in summer than in those assessed in winter $(p=0.0464)$. Radiological findings were not seasonvariable.

Conclusion: Low serum concentration of bone alkaline phosphatase seems to be a risk factor for low bone mineral density and, by extension, for osteoporosis. Therefore it should be considered as an important laboratory test in JIA patients suspected of secondary osteoporosis. Clinical significance of osteoprotegerin and Beta-Crosslaps needs to be furthe investigated.

Disclosure of Interests: None declared

DOI: 10.1136/annrheumdis-2019-eular.1867

\section{THU0549 SURGICAL MANAGEMENT IN JUVENILE IDIOPATHIC ARTHRITIS: A MONOCENTRIC EXPERIENCE OF 257 PROSTHESIS}

Irene Pontikaki ${ }^{1}$, Carolina Artusi ${ }^{2}$, Marco DI Marco ${ }^{3}$, Alessandro Sinelli $^{2}$, Martina Cornalba ${ }^{3}$, Marcello Truzzi ${ }^{3}$, Silvia De Martinis ${ }^{3}$, Roberto Viganò ${ }^{3}$. ${ }^{1} A S S T$ Pini - CTO, Unit Of Pediatric Rheumatology-Department Of Rheumatology, Milan, Italy; ${ }^{2}$ ASST Pini - CTO, Unit Of Rheumatology, Milan, Italy, ${ }^{3}$ ASST Pini - CTO,

Unit of Rheuma Surgery, Milan, Italy

Background: Juvenile idiopathic arthritis (JIA) includes all forms of inflammatory arthritis, with onset before the $16^{\text {th }}$ birthday, lasting more than 6 weeks. Although conservative management with nonsteroidal anti-inflammatory drugs and disease-modifying antirheumatic drugs can be effective, a significant number of young adults affected by JIA requires a major surgical procedure, including different types of prosthetic devices.

Objectives: This article presents a monocentric experience of an interdisciplinary team of rheumatologists and orthopedic surgeons for JIA and the outcome of patients subjected to prosthesis implantation.

Methods: Data were collected through a retrospective analysis of 583 patients attending to our transitional care center for JIA between 1999 and 2018, in the context of a once-week rheumatologic counseling with the orthopedic surgeons. Descriptive statistics were used to evaluate the baseline and follow-up data

Results: On a total of 257 prosthesis implantations, 137 were total hip prosthesis, 101 total knee prosthesis and 19 total ankle prosthesis. The 10 -year implant survival rate was $97 \%$, with good results in term of function and comfort for the patients. Complications were observed in $9 \%$ of 